\title{
A COMBUSTÃo CATALÍTICA DO METANO: ESTUdO ESTATÍSTICO DO EFEITO DAS VARIÁVEIS DE PREPARAÇÃO E PRÉ-TRATAMENTO DE CATALISADORES DE PALÁDIO SUPORTADO SOBRE A ATIVIDADE CATALÍTICA
}

\author{
Maria da Graça Carneiro da Rocha* \\ Departamento de Físico-Química, Instituto de Química, UFBA, Campus de Ondina, 40170-290 Salvador - BA \\ Roger Frety \\ Laboratoire des Applications de la Chimie à l'Environnement, LACE/CNRS, Lyon, France \\ Pierre Lanteri \\ CPE, Ecole Supérieure de Chimie Physique Electronique de Lyon, France
}

Recebido em 2/8/99; aceito em 10/10/00

\begin{abstract}
THE CATALYTIC COMBUSTION OF METHANE: STATISTICAL STUDY OF PREPARATION AND PRETREATMENT CONDITIONS OF PALLADIUM SUPPORTED CATALYSTS AND THEIR RELATIONSHIP WITH CATALYTIC ACTIVITY. The catalytic combustion of methane on alumina supported palladium catalysts was studied. It has been reported that the activity of the catalyst increases with its time on line, despite of an increase of the palladium particle size. However, different preparation, pretreatment and testing conditions can be the reason for the observed different results. An experimental design, which allows to verify the influence of several parameters at the same time with a good statistical quality, was used. A Plackett-Burman design was selected for the screening of the variables which have an effect on the increase of the catalyst activity.
\end{abstract}

Keywords: catalytic combustion of methane; supported palladium catalyst; experimental design.

\section{INTRODUÇÃO}

A combustão catalítica do metano tem sido estudada nos últimos anos em vista da sua aplicação na área de controle ambiental. O metano é o hidrocarboneto mais difícil de ser oxidado e a reação em fase homogênea ocorre normalmente a temperaturas superiores a $900^{\circ} \mathrm{C}$. A queima do metano é também importante do ponto de vista da geração de energia, para aquecimento em pequena escala e em turbinas a gás. Do ponto de vista fundamental esta reação tem despertado também bastante interesse.

Os catalisadores de metal nobre suportados são muito ativos para a reação de oxidação total do metano em especial o $\mathrm{Pd}$ e a $\mathrm{Pt}^{1}$. Diversos estudos têm sido realizados utilizando catalisadores de Pd e Pt suportados ${ }^{2-9}$. Utilizando-se diferentes catalisadores e condições de preparação: metais, tipos de suportes, precursores e pré-tratamento (vide Tabela 1), observou-se que a atividade catalítica aumentava após um ciclo de reação a tempo e temperatura variáveis. Esta ativação do catalisador de paládio em meio de reação, foi observada por diversos autores ${ }^{2-11}$, trabalhando em condições diferentes entre si, e foi atribuída a diversas causas como à capacidade de adsorção do oxigênio sobre o metal ${ }^{2}$, à morfologia do catalisador $^{3,4}$, à dispersão do óxido de paládio sobre os cristalitos de paládio ${ }^{5,6}$.

A fim de verificar se o aumento de atividade de catalisadores de paládio é um fenômeno geral que se produz cada vez que o catalisador é exposto ao meio reacional, selecionou-se para avaliação uma série de variáveis de preparação e de prétratamento do catalisador. O objetivo deste trabalho é verificar se a ativação do catalisador ocorre devido à presença do meio reacional (metano, oxigênio, dióxido de carbono e água) e quais as variáveis de preparação e pré-tratamento do catalisador associadas a esta ativação.

\footnotetext{
*e-mail: mgcr@ufba.br
}

\section{PARTE EXPERIMENTAL}

Para este estudo escolheu-se uma metodologia de plano estatístico de experimentos, que permite estudar simultaneamente vários fatores com a melhor qualidade estatística sobre a informação obtida. Esta metodologia permite um crivo (screening) das variáveis. Utilizando uma matriz de Hadamard (ou plano de Plackett-Burman) é possível distinguir entre os fatores de alta e média influência e sem efeito sobre uma determinada medida, neste caso específico, a velocidade da reação de combustão do metano. O primeiro passo é selecionar as variáveis que podem ter efeito sobre a atividade catalítica: variáveis de preparação do catalisador e condições da reação catalítica.

Tendo em vista o grande número de variáveis a serem levadas em conta na preparação e pré-tratamento dos catalisadores, algumas foram mantidas constantes: a natureza do metal e do precursor ( $\mathrm{Pd}$ e cloreto de paládio, respectivamente), o método de preparação (impregnação úmida), a atmosfera de pós-tratamento do catalisador (tratamento em meio da combustão catalítica do metano por 3 horas). O procedimento experimental foi normalizado e os catalisadores submetidos a diferentes pré-tratamentos foram testados em condições padrão (temperatura de $320^{\circ} \mathrm{C}$ durante 4 horas).

As variáveis $\left(\mathrm{X}_{\mathrm{i}}\right)$ consideradas estão resumidas nas Tabelas 2 e 3 a seguir.

Para este número de variáveis utilizando-se a matriz de Hadamard H8, 8 experimentos são necessários. Neste caso, para estimar o "peso" destas 6 variáveis de forma independente e com a maior precisão possível, postula-se um polinômio da forma:

$\mathrm{Y}=\mathrm{b}_{0}+\Sigma \mathrm{b}_{\mathrm{i}} \mathrm{X}_{\mathrm{i}}$

onde $b_{i}$ são os pesos dos fatores $X_{i}$ expressos como -1 e +1 .

A substituição das variáveis codificadas (Tabela 4) pelos seus valores reais forneceu o plano de experimentos da Tabela 5. A coluna $X_{6}$ apresenta ainda os códigos $(-)$ e $(+)$, pois 
Tabela 1. Oxidação catalítica do metano - Resumo da literatura.

\begin{tabular}{|c|c|c|c|c|c|c|c|c|c|}
\hline \multicolumn{4}{|c|}{ Catalisador } & \multicolumn{2}{|c|}{ Pré-tratamento } & \multirow[t]{2}{*}{ Ativação } & \multirow{2}{*}{\multicolumn{2}{|c|}{$\begin{array}{l}\text { Reação } \\
\mathrm{O}_{2} / \mathrm{CH}_{4}\end{array}$}} & \multirow[t]{2}{*}{ Ref } \\
\hline$\%$ & metal & suporte & precursor & gás & $\begin{array}{l}\text { Temp. } \\
\left({ }^{\circ} \mathrm{C}\right)\end{array}$ & & & & \\
\hline $2,7-40$ & $\mathrm{Pd}$ & $\begin{array}{c}\mathrm{TiO}_{2} \\
\gamma-\mathrm{Al}_{2} \mathrm{O}_{3} \\
\alpha-\mathrm{Al}_{2} \mathrm{O}_{3} \\
\mathrm{ThO}_{2} \\
\mathrm{SnO}_{2}\end{array}$ & $\begin{array}{l}\mathrm{Pd}\left(\mathrm{NH}_{3}\right)_{4} \mathrm{Cl}_{2} \\
\mathrm{Pd} \text { óxido(II) } \\
\mathrm{Pd} \text { óxido(IV) }\end{array}$ & $\begin{array}{c}\text { He ou } \mathrm{H}_{2} \\
\mathrm{O}_{2}\end{array}$ & 502 & não & - & $\begin{array}{c}0,45 / 1+\mathrm{He} \\
2 / 1+\mathrm{He}\end{array}$ & 2 \\
\hline $2,7-10$ & $\mathrm{Pt}$ & $\begin{array}{c}\mathrm{TiO}_{2} \\
\gamma-\mathrm{Al}_{2} \mathrm{O}_{3} \\
\mathrm{ThO}_{2}\end{array}$ & $\mathrm{Pt}\left(\mathrm{NH}_{3}\right)_{4} \mathrm{Cl}_{2}$ & $\begin{array}{c}\text { He ou } \mathrm{H}_{2} \\
\mathrm{O}_{2}\end{array}$ & 502 & não & - & $0,45 / 1$ & 2 \\
\hline $0,5-2,0$ & $\mathrm{Pt}$ & & $\begin{array}{c}\mathrm{H}_{2} \mathrm{PtCl}_{6} \\
\operatorname{Pt}\left(\mathrm{NH}_{3}\right)_{4} \mathrm{Cl}_{2}\end{array}$ & ar & 500 & $\operatorname{sim}$ & $1-17 \mathrm{~h}$ & $2,2 / 1+\mathrm{He}$ & 5 \\
\hline $0,2-2,3$ & $\mathrm{Pd}$ & $\begin{array}{c}\alpha-\mathrm{Al}_{2} \mathrm{O}_{3} \\
\gamma-\mathrm{Al}_{2} \mathrm{O}_{3} \\
\mathrm{ZrO}_{2}\end{array}$ & $\begin{array}{c}\mathrm{Pd}\left(\mathrm{NH}_{3}\right)_{4} \mathrm{Cl}_{2} \\
\mathrm{H}_{2} \mathrm{PdCl}_{4}\end{array}$ & ar & $\begin{array}{l}500 \\
700\end{array}$ & $\operatorname{sim}$ & $1-8 \mathrm{~h}$ & $2,2 / 1+\mathrm{He}$ & 5 \\
\hline $2,2-2,3$ & $\mathrm{Pd}$ & $\gamma-\mathrm{Al}_{2} \mathrm{O}_{3}$ & $\mathrm{H}_{2} \mathrm{PdCl}_{4}$ & ar & $\begin{array}{l}500 \\
700 \\
900\end{array}$ & $\operatorname{sim}$ & - & $2,2 / 1+\mathrm{He}$ & 6 \\
\hline $\begin{array}{c}1-5 \\
5\end{array}$ & $\begin{array}{l}\mathrm{Pd} \\
\mathrm{Pd}\end{array}$ & $\begin{array}{l}\delta-\mathrm{Al}_{2} \mathrm{O}_{3} \\
\gamma-\mathrm{Al}_{2} \mathrm{O}_{3}\end{array}$ & $\begin{array}{l}\mathrm{H}_{2} \mathrm{PdCl}_{4} \\
\mathrm{H}_{2} \mathrm{PdCl}_{4} \\
\mathrm{Pd}\left(\mathrm{NO}_{3}\right)\end{array}$ & $\begin{array}{l}\text { ar } \\
\mathrm{O}_{2} \\
\mathrm{H}_{2}\end{array}$ & $\begin{array}{c}500-850 \\
400-600 \\
400\end{array}$ & $\begin{array}{l}\operatorname{sim} \\
\operatorname{sim}\end{array}$ & $\stackrel{-}{\text { minutos-dias }}$ & $\begin{array}{l}\text { ar/1\% } \mathrm{CH}_{4} \\
\text { ar/1\% } \mathrm{CH}_{4}\end{array}$ & $\begin{array}{l}3 \\
4\end{array}$ \\
\hline $\begin{array}{l}1,95 \\
1,95 \\
1,96 \\
2,18 \\
2,16\end{array}$ & $\begin{array}{l}\mathrm{Pd} \\
\mathrm{Pt} \\
\mathrm{Pd} \\
\mathrm{Pd} \\
\mathrm{Pd}\end{array}$ & $\begin{array}{c}\delta+\gamma-\mathrm{Al}_{2} \mathrm{O}_{3} \\
\delta+\gamma-\mathrm{Al}_{2} \mathrm{O}_{3} \\
\mathrm{Al}_{2} \mathrm{O}_{3} \\
\mathrm{Al}_{2} \mathrm{O}_{3} \\
\mathrm{SiO}_{2}\end{array}$ & $\begin{array}{c}\mathrm{H}_{2} \mathrm{PdCl}_{4} \\
\mathrm{H}_{2} \mathrm{PtCl}_{6} \\
\mathrm{H}_{2} \mathrm{PdCl}_{4} \\
\text { acetilacetonato } \\
\mathrm{Pd}\left(\mathrm{NH}_{3}\right)_{4}\end{array}$ & $\begin{array}{c}\mathrm{N}_{2}+\mathrm{H}_{2} \\
\mathrm{~N}_{2}+\mathrm{H}_{2} \\
\mathrm{H}_{2} \\
\mathrm{H}_{2} \\
\mathrm{H}_{2}\end{array}$ & $\begin{array}{l}500 \\
500 \\
400 \\
400 \\
400\end{array}$ & $\begin{array}{l}\text { sim } \\
\text { sim } \\
\text { sim } \\
\text { sim } \\
\text { não }\end{array}$ & $\begin{array}{l}- \\
- \\
- \\
-\end{array}$ & $\begin{array}{l}4 / 1 \\
4 / 1 \\
4 / 1 \\
4 / 1 \\
4 / 1\end{array}$ & $\begin{array}{c}8 \\
14 \\
11\end{array}$ \\
\hline $\begin{array}{c}8,5 \\
1-10 \\
0,77-7,7\end{array}$ & $\begin{array}{l}\mathrm{Pd} \\
\mathrm{Pd} \\
\mathrm{Pd}\end{array}$ & $\begin{array}{l}\mathrm{Al}_{2} \mathrm{O}_{3} \\
\mathrm{ZrO}_{2}\end{array}$ & $\begin{array}{c}\mathrm{PdCl}_{2} \\
\mathrm{Pd}\left(\mathrm{NH}_{3}\right) \mathrm{NO}_{2} \\
\mathrm{Pd}\left(\mathrm{NH}_{3}\right) \mathrm{NO}_{2}\end{array}$ & $\begin{array}{l}\text { ar } \\
\text { ar } \\
\text { ar }\end{array}$ & $\begin{array}{l}850 \\
850 \\
850\end{array}$ & $\begin{array}{l}\operatorname{sim} \\
\operatorname{sim} \\
\operatorname{sim}\end{array}$ & $\begin{array}{l}- \\
- \\
-\end{array}$ & $\begin{array}{l}\text { ar/2\% } \mathrm{CH}_{4} \\
\text { ar/2\% } \mathrm{CH}_{4} \\
\text { ar/2\% } \mathrm{CH}_{4}\end{array}$ & 9 \\
\hline
\end{tabular}

Tabela 2. Domínio experimental das variáveis $\left(X_{i}\right)$ estudadas.

\begin{tabular}{|c|c|c|}
\hline \multirow[t]{2}{*}{ Variáveis } & \multicolumn{2}{|c|}{ Níveis } \\
\hline & $(-)$ & $(+)$ \\
\hline $\mathrm{X}_{1}$-suporte $\left(\mathrm{Al}_{2} \mathrm{O}_{3}\right)$ & alfa & delta \\
\hline $\mathrm{X}_{2}$-teor $\mathrm{Pd}$ & $0,5 \%$ & $3 \%$ \\
\hline $\mathrm{X}_{3}$-temp. pré-calcinação & $400^{\circ} \mathrm{C}$ & $700^{\circ} \mathrm{C}$ \\
\hline $\mathrm{X}_{4}$-temp. pré-redução & $400^{\circ} \mathrm{C}$ & $800^{\circ} \mathrm{C}$ \\
\hline $\mathrm{X}_{5}$-Razão $\mathrm{O}_{2} / \mathrm{CH}_{4}$ & 1/1 (sub-esteq.) & 4/1 (sobre-esteq.) \\
\hline $\mathrm{X}_{6}$-temp. pós-tratamento & $(-1)$ & $(+1)$ \\
\hline
\end{tabular}

Tabela 3. Valores assumidos pela variável $\mathrm{X}_{6}$, temperatura de pós-tratamento, sob meio de reação.

\begin{tabular}{c}
\hline Pós-tratamento sob meio de reação $\left(\mathrm{X}_{6}\right)$ \\
\hline -sem tratamento $(\mathrm{SR})$ \\
-tratamento a $500^{\circ} \mathrm{C}$ \\
-tratamento a $700^{\circ} \mathrm{C}$ \\
-tratamento a $900^{\circ} \mathrm{C}$ \\
\hline
\end{tabular}

foram comparados os quatro tratamentos (Tabela 3) dois a dois:

1. Sem tratamento (-) comparado a tratamento em meio de reação à temperatura de $900^{\circ} \mathrm{C} \mathrm{(+)}$

2. Sem tratamento (-) comparado a tratamento em meio de reação à temperatura de $500^{\circ} \mathrm{C}(+)$

3. Tratamento em meio de reação a $700^{\circ} \mathrm{C}(-)$ comparado ao tratamento em meio de reação a $900^{\circ} \mathrm{C}(+)$
4. Tratamento em meio de reação a $500^{\circ} \mathrm{C}(-)$ comparado ao tratamento em meio de reação a $900^{\circ} \mathrm{C}(+)$

Tabela 4. Estrutura da matriz de Hadamard H8 para sistema com 6 variáveis.

\begin{tabular}{ccccccc}
\hline Experiência & $\mathrm{X}_{1}$ & $\mathrm{X}_{2}$ & $\mathrm{X}_{3}$ & $\mathrm{X}_{4}$ & $\mathrm{X}_{5}$ & $\mathrm{X}_{6}$ \\
\hline 1 & + & + & + & - & + & - \\
2 & - & + & + & + & - & + \\
3 & - & - & + & + & + & - \\
4 & + & - & - & + & + & + \\
5 & - & + & - & - & + & + \\
6 & + & - & + & - & - & + \\
7 & + & + & - & + & - & - \\
8 & - & - & - & - & - & - \\
\hline
\end{tabular}

Tabela 5. Plano de experimentos-Variáveis naturais estudadas.

\begin{tabular}{lcccccc}
\hline $\begin{array}{l}\text { Experiência } \\
\mathrm{N}^{\circ} .\end{array}$ & $\begin{array}{c}\mathrm{U} 1 \\
\text { Alumina }\end{array}$ & $\begin{array}{c}\mathrm{U} 2 \\
\% \mathrm{Pd}\end{array}$ & $\begin{array}{c}\mathrm{U} 3 \\
\mathrm{~T}_{\text {red }} \\
\left({ }^{\circ} \mathrm{C}\right)\end{array}$ & $\begin{array}{c}\mathrm{U} 4 \\
\mathrm{~T}_{\text {calc }} \\
\left({ }^{\circ} \mathrm{C}\right)\end{array}$ & $\begin{array}{c}\mathrm{U} 5 \\
\mathrm{O}_{2} / \mathrm{CH}_{4}\end{array}$ & $\begin{array}{c}\mathrm{U} 6 \\
\mathrm{~T}_{\text {post }} \\
\left({ }^{\circ}\right)\end{array}$ \\
\hline 1 & Delta & 3 & 800 & 400 & 4 & -1 \\
2 & Alfa & 3 & 800 & 700 & 1 & +1 \\
3 & Alfa & 0,5 & 800 & 700 & 4 & -1 \\
4 & Delta & 0,5 & 400 & 700 & 4 & +1 \\
5 & Alfa & 3 & 400 & 400 & 4 & +1 \\
6 & Delta & 0,5 & 800 & 400 & 1 & +1 \\
7 & Delta & 3 & 400 & 700 & 1 & -1 \\
8 & Alfa & 0,5 & 400 & 400 & 1 & -1 \\
\hline
\end{tabular}


Foram preparados 8 catalisadores e determinada a atividade catalítica, expressa em mol $\mathrm{CH}_{4} / \mathrm{h}$.mol Pd. Os testes catalíticos foram realizados em reator integral contínuo de leito fixo com análise "on-line" por cromatografia gasosa utilizando dois cromatógrafos em série, com detetores TCD e FID, à pressão atmosférica e vazão total de gases de $6 \mathrm{l} / \mathrm{h}$.

Os catalisadores eram preparados por impregnação úmida da alumina, pré-calcinados sob fluxo de nitrogênio e pré-reduzidos sob hidrogênio nas temperaturas indicadas no plano de experimentos, purgados com nitrogênio à temperatura ambiente e armazenados em dessecador. No reator, $1 \mathrm{~g}$ de catalisador era submetido a tratamento sob mistura de reação a 500, 700 e $900^{\circ} \mathrm{C}$ durante $3 \mathrm{~h}$, após o que era resfriado à temperatura de $320^{\circ} \mathrm{C}$ para o teste de atividade catalítica (4 h de duração).

O primeiro passo neste estudo foi preparar um catalisador de referência seguindo procedimento descrito na literatura ${ }^{7,8}$ com o objetivo de verificar a ativação do catalisador de paládio suportado em alumina no meio de reação. Para tal foi preparado um catalisador contendo $1,88 \%$ de paládio suportado sobre alumina de transição SCM-129 da Rhône-Poulenc. Este catalisador foi pré-tratado seguindo o mesmo procedimento experimental utilizado para a série de catalisadores testados no plano estatístico de experimentos, acima descrito. O precursor de todos os catalisadores foi o cloreto de paládio Aldrich e os suportes utilizados no plano de experimentos foram a aluminadelta Puralox SCC 5/90 da Condea e a alumina-alfa SPH 512 da Rhône-Poulenc.

Os catalisadores foram caracterizados por microscopia eletrônica de transmissão (MET), por titulação $\mathrm{O}_{2}-\mathrm{H}_{2}(\mathrm{HT})$ e por difração de raios-X (DRX). A microscopia foi realizada em aparelho JEOL 100CX em observação direta da amostra depositada em grelha de cobre recoberta por um filme de carbono. A titulação $\mathrm{O}_{2}-\mathrm{H}_{2}$ foi realizada em um sistema dinâmico com detetor catarométrico. Para a difração de raios-X utilizou-se um

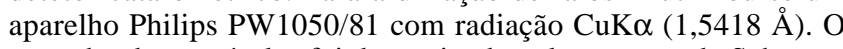
tamanho das partículas foi determinado pela equação de Scherrer.

\section{RESULTADOS}

O efeito do tratamento do catalisador de referência no meio de reação a diferentes temperaturas é mostrado na Figura 1. Confirmando os resultados observados na literatura, observase a ativação do catalisador, com uma diferença de atividade de 24 vezes entre o catalisador simplesmente reduzido e aquele tratado a $900^{\circ} \mathrm{C}$ em meio de reação. Considerando que a fase ativa dos catalisadores sofreu considerável sinterização após tratamento a alta temperatura, como foi evidenciado por técnicas analíticas como microscopia eletrônica de transmissão $^{18}$ (MET), titulação $\mathrm{O}_{2}-\mathrm{H}_{2}{ }^{18}(\mathrm{HT})$ e difração de raios- $\mathrm{X}^{16}$ (DRX), a medida do TOF apresenta uma variação de 90 vezes entre o catalisador antes e após ativação em meio de reação.

\section{Cunversào $(\%)$}

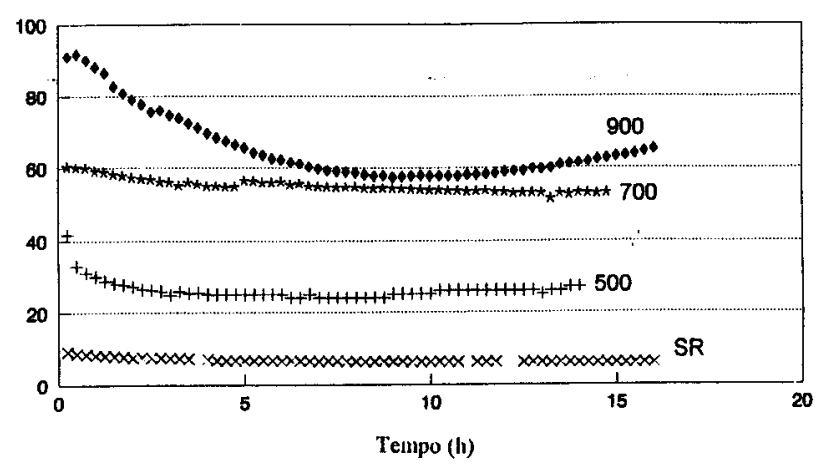

Figura 1. Curvas de conversão a $350^{\circ} \mathrm{C}$ em função do tempo de reação e da temperatura de pós-tratamento, de um catalisador de referência $1,88 \% \mathrm{Pd} / \gamma+\delta-\mathrm{Al}_{2} \mathrm{O}_{3}(\mathrm{SCM} 129)$.
Os resultados dos testes de atividade catalítica para os catalisadores preparados e testados segundo o plano estatístico de experimentos são apresentados na Tabela 6 , onde podem ser observados valores de atividade variando entre 0,01 a $74,85 \mathrm{~mol} \mathrm{CH}_{4} /$ h.mol Pd, indicando que existe ao menos um fator de influência sobre a resposta no domínio experimental estudado.

Os coeficientes $b_{i}$ calculados são apresentados na Tabela 7 e representados na Figura 2 (a) a (d).

Tabela 6. Atividades finais a $320^{\circ} \mathrm{C}\left(\mathrm{mol} \mathrm{CH}_{4} / \mathrm{h} . \mathrm{mol} \mathrm{Pd}\right)$.

\begin{tabular}{ccccc}
\hline Experiência & $\begin{array}{c}\text { Sem } \\
\text { tratamento }\end{array}$ & $\begin{array}{c}\text { Tratam. } \\
\text { a } 500^{\circ} \mathrm{C}\end{array}$ & $\begin{array}{c}\text { Tratam. } \\
\text { a } 700^{\circ} \mathrm{C}\end{array}$ & $\begin{array}{c}\text { Tratam. } \\
\text { a } 900^{\circ} \mathrm{C}\end{array}$ \\
\hline 1 & 0,63 & 4,81 & 10,90 & 8,35 \\
2 & 0,05 & 0,11 & 0,76 & 1,04 \\
3 & 0,16 & 0,12 & 0,01 & 0,14 \\
4 & 1,87 & 13,62 & 74,85 & 21,74 \\
5 & 2,75 & 2,48 & 4,98 & 3,44 \\
6 & 0,32 & 4,21 & 15,07 & 16,73 \\
7 & 0,39 & 0,48 & 1,77 & 1,69 \\
8 & 0,11 & 1,84 & 1,05 & 1,28 \\
\hline
\end{tabular}

Tabela 7. Valores dos efeitos obtidos: coeficientes " $b_{i}$ ".

\begin{tabular}{lcccc}
\hline Coeficiente $\left(\mathrm{b}_{\mathrm{i}}\right)$ & $\begin{array}{c}\text { Sem } \\
\text { trat./900 }\end{array}$ & $\begin{array}{c}\text { Sem } \\
\text { trat./500 }\end{array}$ & $\begin{array}{c}700 / \\
900\end{array}$ & $\begin{array}{c}500 / \\
900\end{array}$ \\
\hline $\mathrm{b}_{0}$ (efeito médio) & 5,53 & 2,71 & 7,08 & 6,28 \\
$\mathrm{~b}_{1}$ (suporte) & 4,34 & 2,00 & 5,70 & 4,67 \\
$\mathrm{~b}_{2}(\% \mathrm{Pd})$ & $-4,16$ & $-1,81$ & $-2,80$ & $-3,83$ \\
$\mathrm{~b}_{3}\left(\mathrm{~T}_{\text {red }}\right)$ & $-0,89$ & $-1,44$ & 0,08 & $-0,60$ \\
$\mathrm{~b}_{4}\left(\mathrm{~T}_{\text {calc }}\right)$ & 0,30 & 0,86 & $-0,94$ & $-0,43$ \\
$\mathrm{~b}_{5}\left(\mathrm{O}_{2} / \mathrm{CH}_{4}\right)$ & 0,96 & 1,51 & 1,94 & 1,25 \\
$\mathrm{~b}_{6}\left(\mathrm{~T}_{\text {pos-trat }}\right)$ & 5,16 & 2,39 & 3,65 & 4,46 \\
\hline
\end{tabular}

\section{DISCUSSÃO}

Em um estudo estatístico, diversas "respostas" podem ser escolhidas em função do fenômeno que está sendo avaliado. A atividade catalítica $\left(\mathrm{mol} \mathrm{CH}_{4} / \mathrm{h}\right.$. mol Pd) é significativa pois evidencia que o catalisador quando exposto ao meio de reação torna-se efetivamente mais ativo, mesmo havendo sido submetido a altas temperaturas que provocaram uma sinterização da fase ativa. Análises por DRX ${ }^{16}$ demonstraram que ambos os suportes mantiveram-se estáveis nas condições de teste.

Como se pode observar na Tabela 7 e nas Figuras 2 (a) a 2 (d) o tipo de suporte $\left(\mathrm{X}_{1}\right)$ tem uma influência primordial sobre a atividade catalítica. Outros fatores são o teor em metal $\left(\mathrm{X}_{2}\right)$ e o tratamento em meio reacional $\left(\mathrm{X}_{6}\right)$ à mais alta temperatura. A comparação do catalisador simplesmente reduzido com aquele tratado à temperatura de $900^{\circ} \mathrm{C}$ em meio de reação revela a importante influência deste tratamento. Assim, a atividade catalítica aumenta quando é utilizada a alumina de transição $\left(b_{1}=5,53\right)$, sendo favorecida pelo baixo teor de paládio $\left(b_{2}=\right.$ $-4,16)$. $\mathrm{O} \mathrm{b}_{6}=5,16$ significa que a alta atividade está relacionada à maior temperatura de tratamento em meio reacional. Os demais fatores não parecem ter influência.

Os catalisadores novos e após os diferentes pré-tratamentos foram caracterizados por diversas técnicas visando determinar as modificações sofridas pelo sítio ativo e consequentemente a causa da ativação comprovada pelo plano de experimentos. A Tabela 8 de medidas de tamanho de partícula obtidas por titulação $\mathrm{O}_{2}-\mathrm{H}_{2}{ }^{17}(\mathrm{HT})$, microscopia eletrônica de transmissão (MET) e difração de raios-X (DRX) para o catalisador de referência $1,88 \% \mathrm{Pd} / \mathrm{Al}_{2} \mathrm{O}_{3}$ mostra que ocorreu sinterização, que também foi observada em todos os catalisadores testados. No entanto, somente estas medidas não explicam a ativação pois catalisadores com semelhantes dispersões metálicas apresenta- 


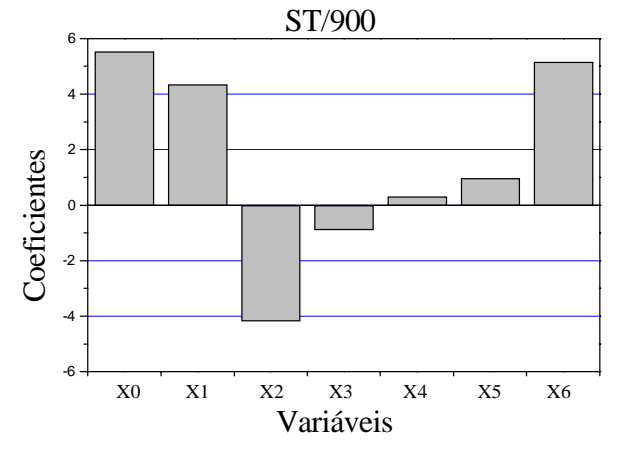

(a)

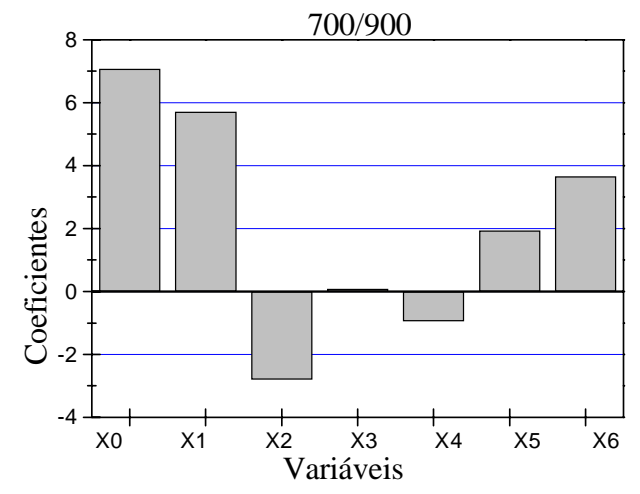

(b)

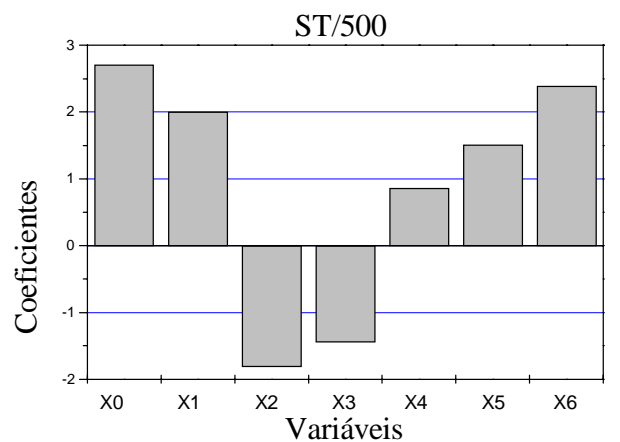

(c)

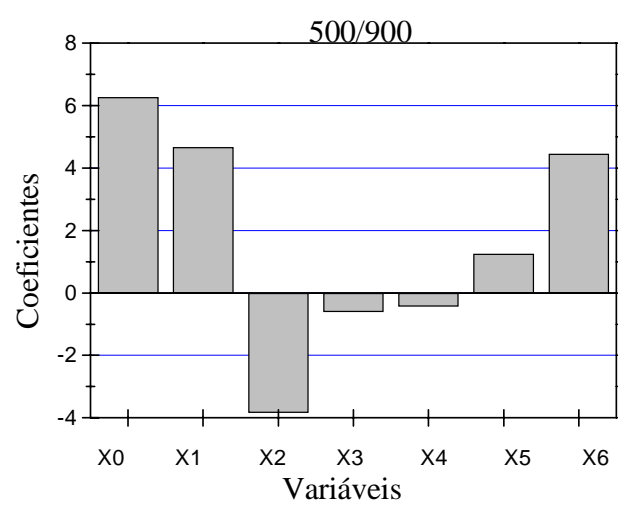

(d)

Figura 2. Influência do tratamento: a) sem tratamento comparado ao tratamento em meio reacional a $900^{\circ} \mathrm{C}$, b) sem tratamento comparado ao tratamento em meio reacional a $500^{\circ} \mathrm{C}$, c) efeito da temperatura de pré-tratamento em meio reacional (700-900 $\left.{ }^{\circ} \mathrm{C}\right)$ e d) efeito da temperatura de pré-tratamento em meio reacional $\left(500-900^{\circ} \mathrm{C}\right)$.

Tabela 8. Tamanho de partículas metálicas $(\AA)$ do catalisador de referência $1,88 \% \mathrm{Pd} / \mathrm{Al}_{2} \mathrm{O}_{3}$.

\begin{tabular}{lcccc}
\hline Catalisador & \multicolumn{2}{c}{ DRX } & MET & HT \\
& PdO & Pd & & \\
\hline $\begin{array}{l}\text { Novo } \\
\text { SR (apenas } \\
\text { reduzido) }\end{array}$ & $(1)$ & $(1)$ & 30 & 25 \\
$\begin{array}{l}\text { Tratado a } 700^{\circ} \mathrm{C}, \\
\text { meio de reação } \\
\text { Tratado a 900 }\end{array}$ & 140 & 280 & $200-700$ & 250 \\
meio de reação & 120 & 290 & $400-700$ & 300 \\
\hline
\end{tabular}

(1) Cristais muito pequenos

ram atividades bem diferentes. Como na reação de oxidação a área metálica não tem relação direta com os sítios ativos durante a reação a sua medida não permite maiores conclusões.

Análises de redução a temperatura programada (TPR), nanodifração, XPS e FTIR mostram que a superfície dos catalisadores é composta por $\mathrm{Pd}$ e $\mathrm{PdO}$, o que efetivamente representa a fase ativa para esta reação ${ }^{18}$.

O plano de experiências mostrou que do tipo do suporte depende a capacidade do catalisador de atingir um estado mais ativo. É provável que o tipo de suporte condicione também o teor ótimo de metal da fase ativa o que foi comprovado em testes posteriores; os catalisadores suportados em alumina de transição com baixo teor de $\mathrm{Pd}(0,5 \%)$ são mais ativos. Os catalisadores suportados sobre alumina alfa mais ativos são aqueles de mais alto teor de Pd (3\%). Em função destes fatos e do que está descrito na literatura ${ }^{2-8}$ sugere-se que de acordo com o tipo de interação existente entre a fase ativa e o suporte, o paládio pode atingir seu estado mais ativo e estável a uma velocidade diferente. Catalisadores suportados em alumina-delta, ao final da preparação, apresentam interação com o suporte. $\mathrm{O}$ catalisador está no estado metálico, recoberto por uma camada de óxido como indicaram as análises de $\mathrm{TPR}^{12,13}$. Ao ser colocado em meio de reação a camada de óxido aprofunda-se e o catalisador passa a conter uma mistura das duas fases. As partículas estando inicialmente muito dispersas sobre o suporte apresentam forte interação. Com a reação, a tendência das partículas é sinterizar e desta forma livrar-se do efeito do suporte e consequentemente, sofrerem ciclos de oxi-redução. Testes de atividade realizados em ausência de oxigênio mostraram que a reação é mantida por um período às custas do oxigênio armazenado no catalisador.

Os catalisadores suportados em alumina-alfa apresentaram atividade inferior aqueles sobre alumina de transição, provavelmente porque nas drásticas condições de pré-tratamento, a fase ativa ligou-se mais fortemente ao suporte. Experimentalmente observou-se que o catalisador $0,5 \% \mathrm{Pd} /$ alfa-alumina foi o menos ativo de toda a série testada o que comprova esta hipótese.

Este fato evidencia-se em outros trabalhos ${ }^{2,3,10}$ com catalisadores suportados em sílica em que o paládio estaria mais livre de interações com o suporte. Parece evidente que os sítios ativos dos catalisadores de $\mathrm{Pd} / \mathrm{Al}_{2} \mathrm{O}_{3}$ não estão prontos antes da reação, mas são formados no seu decorrer.

Os resultados de caracterização corroboram esta hipóte$\mathrm{se}^{12,13,15}$ pois verificou-se que de acordo com o tipo de suporte, o estado inicial dos diversos catalisadores é diferente e que a reação a alta temperatura em meio reacional (ou a reação de longa duração a uma temperatura baixa) conduz a espécies similares. O efeito do suporte é deste modo, liberar mais ou menos facilmente a fase ativa, permitindo atingir um tamanho crítico para manter ciclos permanentes de óxido-redução. Todos os catalisadores foram caracterizados por TPR, DRX, MET dentre outras técnicas que mostraram a evolução das espécies de superfície e mássicas, $\mathrm{Pd}$ e $\mathrm{PdO}$, assim como do tamanho dessas partículas. É importante ressaltar, que como esperado, o 
tratamento a altas temperaturas levou à sinterização das partículas da fase ativa que variou de $1-4 \mathrm{~nm}$ a $100 \mathrm{~nm}$ após tratamento a alta temperatura em meio de reação ${ }^{15}$. O aumento do tamanho da partícula não levou a uma perda da atividade catalítica, pelo contrário. Análises da superfície indicam a presença de $\mathrm{PdO}$ e Pd. Estes resultados estão de acordo com McCarty ${ }^{10}$ que atribui as diferenças observadas na taxas de reação à formação de espécies oxidadas superficiais cujas características dependem da morfologia das espécies paládio metálico e óxido de paládio subjacentes. A oxidação do paládio seria por sua vez dependente da temperatura e do suporte. Por sua vez, Fujimoto e colegas ${ }^{19}$ vão mais além ao proporem um mecanismo que relaciona a atividade catalítica para catalisadores de $\mathrm{Pd} / \mathrm{ZrO}_{2}$ às variações de densidade e estabilidade de vacâncias de oxigênio em espécies $\mathrm{PdO}_{\mathrm{x}}$ superficiais. Estas variações seriam dependentes do tamanho do cristalito, da temperatura e do tratamento sob atmosfera gasosa específica.

\section{CONCLUSÕES}

A análise do plano estatístico de experimentos mostrou que, independentemente das diferentes condições de preparação e prétratamento do catalisador, como descrito na literatura ${ }^{2,9}$, o meio de reação é um fator primordial para a ativação de catalisadores de $\mathrm{Pd} / \mathrm{Al}_{2} \mathrm{O}_{3}$. Também têm influência sobre a atividade catalítica: o tipo de suporte, o teor em metal e a temperatura de tratamento em meio de reação. Os fatores favoráveis para a ativação são a alumina de transição, o baixo teor de metal $(0,5 \%)$ e a mais alta temperatura de tratamento em meio de reação, entre 700 e $900^{\circ}$ $C$, apesar da sinterização da fase ativa inerente a este tratamento. Análises de caracterização realizadas permitiram verificar que o sítio ativo é composto por paládio metálico e óxido de paládio.

\section{AGRADECIMENTOS}

Maria da Graça Carneiro da Rocha agradece ao CNPq pelo apoio financeiro.

\section{REFERÊNCIAS}

1. Trimm, D. L.; Lam, C. W.; Chem. Eng. Sci. 1980, $35,1405$.

2. Cullis, C. F.; Willatt, B. M.; J. Catal. 1983, 83, 267.

3. Baldwin, T. R.; Burch, R.; Appl. Catal. 1990, 66, 337.

4. Baldwin, T. R.; Burch, R.; Appl. Catal. 1990, 66, 359.

5. Hicks, R. F.; Qi, H.; Young, M. L.; Lee, R. G.; J. Catal. 1990, 122, 280.

6. Hicks, R. F.; Qi, H.; Young, M. L.; Lee, R. G.; J. Catal. 1990, 122, 295.

7. Briot, P., Tese de Doutorado, Universidade Claude Bernard-Lyon I, França, 1991.

8. Briot, P.; Primet, M.; Appl. Catal. 1991, 68, 301.

9. Ribeiro, F. H.; Chow, M.; Dalla-Betta, R.A.; J. Catal. 1994, 146, 537.

10. McCarty, J. G.; Catal. Today 1995, 26, 283.

11. Marin, J. L. H., Tese de Doutorado, Universidade Claude Bernard Lyon I, França, 1993.

12. Almeida, M. G. C. R.; Beauchesne, F.; Primet, M.; Frety, R.; Book of Abstracts, Vol. 2, Europacat, 1992.

13. Frety, R.; Almeida, M. G. C. R.; Brun, M.; Délichère, P.; Actas XIV Simpósio Ibero-Americano de Catálise, Vol. 1, 1994.

14. Briot, P.; Auroux, A.; Jones, D.; Primet, M.; Appl. Catal. 1990, 59, 141.

15. Rocha, M. G. C.; Frety, R.; $3^{\text {rd }}$ World Congress on Oxidation catalysis in Studies in surface science and catalysis, Ed. Elsevier, 1997, vol 110, p. 767.

16. Rocha, M. G. C.; Frety, R. ; Bergeret, G. ; Anais do $10^{\circ}$ Congresso Brasileiro de Catálise, 1999, vol. 2, p. 368.

17. Benson, J. E.; Hwang, H.S.; Boudart, M.; J. Catal. 1973, 30, 146.

18. Almeida, M. G. C. R.; Tese de Doutorado, Universidade Claude Bernard-Lyon I, França, 1995.

19. Fujimoto, K.; Ribeiro, F. H.; Avalos-Borja, M.; Iglesia, E; J. Catal. 1998, 179, 431. 